\title{
An Affine Arithmetic-Based Power Flow Algorithm Considering the Regional Control of Unscheduled Power Fluctuation
}

\author{
Lizi Luo $^{1}$, Wei Gu ${ }^{1, *}$, Yonghui Wang ${ }^{2}$ and Chunxi Chen ${ }^{2}$ \\ 1 School of Electrical Engineering, Southeast University, Nanjing 210096, China; luolizi@126.com \\ 2 Ninghai Power Supply Company, Zhejiang Electric Power Company of State Grid, Ningbo 315600, China; \\ yonghui_wang2017@163.com (Y.W.); chencx123@126.com (C.C.) \\ * Correspondence: wgu@seu.edu.cn; Tel.: +86-138-1400-5169
}

Received: 29 September 2017; Accepted: 3 November 2017; Published: 7 November 2017

\begin{abstract}
With consideration of the intermittency of renewable generation and uncertain load, a regional control strategy is presented to smooth the unscheduled power fluctuation in this letter. Then, an affine arithmetic-based modeling method is proposed to describe the unscheduled power tracking characteristic of dispatchable generation resources (DGRs), based on which interval power flow solutions with narrower ranges can be obtained. Finally, the proposed algorithm is applied to a modified IEEE 33-bus distribution system to demonstrate its effectiveness.
\end{abstract}

Keywords: affine arithmetic; distribution system; interval power flow; regional control; unscheduled power fluctuation

\section{Introduction}

With the integration of renewable generation and uncertain load, their randomness and intermittency have become indispensable factors in distribution system power flow analysis [1,2]. Interval arithmetic is a tool frequently used to describe the uncertain variables, and the concept of interval power flow for a radial distribution system was originally proposed in Reference [3]. To reduce the conservatism of interval arithmetic, affine arithmetic has been introduced to power flow analysis [4] and appropriately used in radial distribution systems [5], which keeps track of correlations among different variables during the computational process. However, along with the rapidly developed technology of active distribution networks (ADNs), much research has focus on the effects of dispatchable generation resources (DGRs) on distribution systems [6-11]. Among these research studies, adjusting the outputs of DGRs to compensate the unscheduled power fluctuation is considered to be an essential process in the real-time operation of distribution systems [6-8]. This process is a complement to economic dispatch and has many positive effects, such as decreasing the global communication burden, reducing overall network losses, deferring the upgrade of corresponding equipment, and so on. To the author's knowledge, none of the existing interval power flow studies take into consideration the control of DGRs to compensate unscheduled power fluctuation, and thereby lead to the overestimation of interval power flow solutions.

This letter presents a regional control strategy to smooth the unscheduled power fluctuation in distribution systems, based on which an affine arithmetic-based modeling method is proposed to describe the unscheduled power tracking characteristic of DGRs and thus obtain interval power flow solutions with narrower ranges. An IEEE 33-bus distribution system modified with extra uncertain sources is utilized as a test system, and the comparison of interval power flow solutions demonstrates the contribution of this letter. 


\section{Regional Control of Unscheduled Power Fluctuation}

To reduce the deviation of power flow solutions from the scheduled values, unscheduled power fluctuation is always compensated through nearby DGRs in real-time operation. Thus, the distance from an uncertain source to the corresponding DGR is a key factor that should be considered in delimiting the control region. In this section, breadth-first search is utilized to determine the scope of each control region and a simple example with two DGRs is shown in Figure 1.

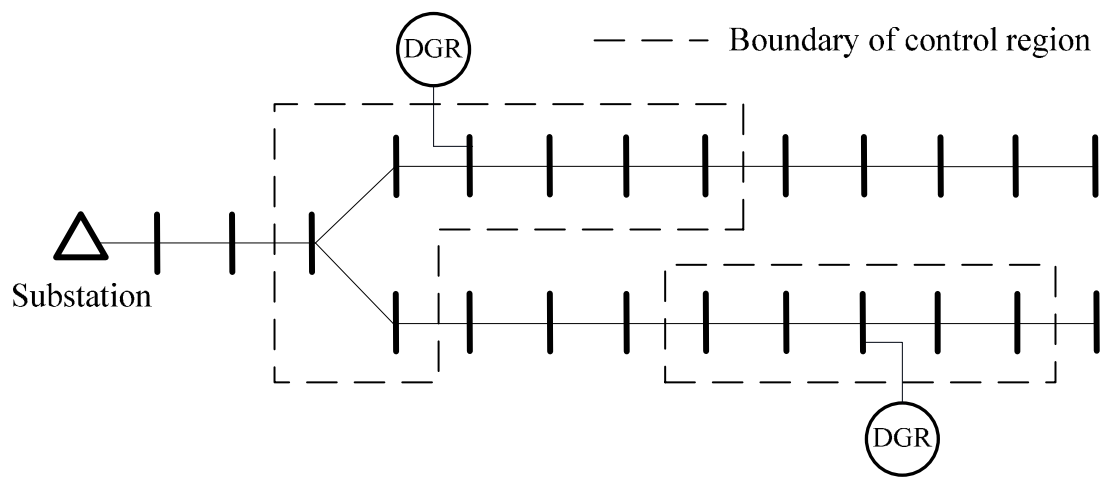

Figure 1. A simple example of control region delimitation.

Each control region is a connected graph, and consists of a DGR as well as several uncertain sources. For any control region $k$, the delimitation of region scope can be described by the equations below:

$$
\begin{gathered}
\sum_{i \in \Omega_{k}^{i n c}} P_{i}^{d e v} \leq P_{k}^{a d j} \quad \forall k=1,2, \cdots, N_{D G R} \\
P_{j}^{d e v}+\sum_{i \in \Omega_{k}^{i n c}} P_{i}^{d e v}>P_{k}^{a d j} \quad \forall j \in \Omega_{k}^{n e i}, \forall k=1,2, \cdots, N_{D G R}
\end{gathered}
$$

where $\Omega_{k}^{i n c}$ indicates the set of buses included in region $k ; \Omega_{k}^{n e i}$ represents the set of neighboring buses of region $k ; N_{D G R}$ is the number of DGRs in the distribution system; $P_{k}^{\text {adj }}$ stands for the adjustable capacity of the DGR in region $k ; P_{i}^{d e v}$ and $P_{j}^{d e v}$ are maximum unscheduled power fluctuations at buses $i$ and $j$, which are equal to the deviations in the corresponding interval models of uncertain sources. Outputs of wind turbines and photovoltaic modules, as well as load demands of electric vehicle charging stations are considered as uncertain sources in this letter, and more details of their interval models can be found in References [12,13]. For any control region, Equation (1) guarantees that the adjustable capacity of the DGR in this region can cover the corresponding unscheduled power fluctuation; Equation (2) shows that adding any neighboring bus to this region would lead to the insufficiency of the DGR's adjustable capacity. Moreover, it is to be noted that buses far from the substation bus are preferential to be included in the control region when several buses with the same distance correspond to the DGR, because a longer power supply distance from the substation bus always leads to more negative impacts.

The scope of each control region varies with the changes of some related factors, which are, respectively, the economic dispatch schedules and the prediction error of uncertain sources. At any time, the target of output control for each DGR is to compensate the total unscheduled power fluctuation in the corresponding region, in order to relieve the impact of power fluctuation on the operation of the power system. To make the description clear, the change of the DGR's output in region $k$ is formulated as:

$$
\Delta P_{k}^{D G R}=-\sum_{i \in \Omega_{k}^{i n c}} \Delta P_{i}^{\text {unsch }}
$$


where $\Delta P_{k}^{D G R}$ represents the change of the DGR's output in region $k$ and $\Delta P_{i}^{\text {unsch }}$ stands for the unscheduled power fluctuation of the uncertain source at bus $i$.

\section{Proposed Affine Arithmetic-Based Modeling Method and Power Flow Algorithm}

At a given time, it is assumed that the active power injection at bus $i$ varies within the range of $\left[\underline{P_{i}}, \overline{P_{i}}\right]$ due to the unscheduled power fluctuation of the connected uncertain source. This power injection can be represented by the affine form:

$$
\stackrel{\wedge}{P}_{i}=\frac{1}{2}\left(\overline{P_{i}}+\underline{P_{i}}\right)+\frac{1}{2}\left(\overline{P_{i}}-\underline{P_{i}}\right) \cdot \varepsilon_{i}
$$

where $\hat{P}_{i}$ stands for the affine form of active power injection at bus $i$ and $\varepsilon_{i}$ is the corresponding noise symbol. Each noise symbol is a symbolic real variable whose value is unknown, except that it is restricted to the interval $[-1,+1]$ and independent from other noise symbols. $\frac{1}{2}\left(\overline{P_{i}}+\underline{P_{i}}\right)$ and $\frac{1}{2}\left(\overline{P_{i}}-\underline{P_{i}}\right)$, respectively, indicate the midpoint and maximum deviation of the active power injection at bus $i$.

To unify the definition of unscheduled power fluctuation, uncertain load demand is regarded as negative power injection. Then the total unscheduled active power fluctuation in region $k$ can be formulated as:

$$
\Delta \hat{P}_{k}^{\text {total }}=\sum_{i \in \Omega_{k}^{\text {inc }}} \frac{1}{2}\left(\overline{P_{i}}-\underline{P_{i}}\right) \cdot \varepsilon_{i}
$$

According to the description in the regional control strategy, the change of the DGR's output in region $k$ can be transformed into the affine form as:

$$
\Delta P_{k}^{\stackrel{D}{D} G R}=-\sum_{i \in \Omega_{k}^{i n c}} \frac{1}{2}\left(\overline{P_{i}}-\underline{P_{i}}\right) \cdot \varepsilon_{i}
$$

Taking into consideration the economic dispatch schedule of the DGR, then the actual output of the DGR is shown in (7):

$$
P_{k}^{\stackrel{\wedge}{D G R}}=P_{k}^{D G R-s c h}-\sum_{i \in \Omega_{k}^{i n c}} \frac{1}{2}\left(\overline{P_{i}}-\underline{P_{i}}\right) \cdot \varepsilon_{i}
$$

where $P_{k}^{D G R-s c h}$ is the scheduled output of the DGR in region $k$. It should be noted that only active power fluctuation is considered in this letter, since most of the reactive power in a distribution system is provided by local reactive power compensation devices (e.g., shunt capacitor and Static Var Compensator), whose outputs are controllable and without uncertainties.

Based on the established affine models of uncertain sources and DGRs in Equations (4) and (7), a backward / forward sweep-based interval power flow algorithm is used to carry out the interval power flow solutions. Details of the utilized interval power flow algorithm can be found in Reference [5]. Figure 2 presents the flowchart of the proposed algorithm. 


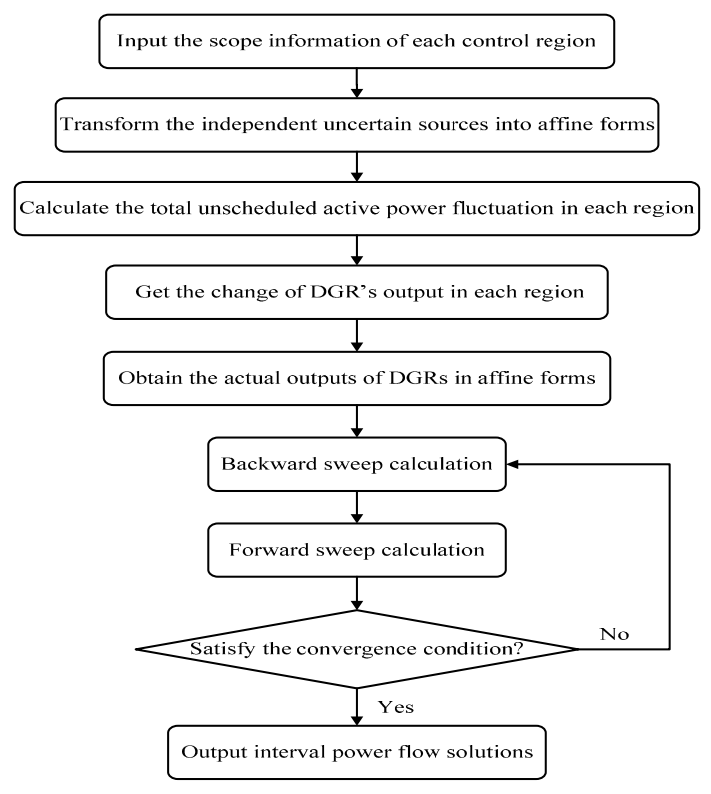

Figure 2. Flowchart of the proposed algorithm.

\section{Case Study}

To demonstrate the positive significance of the proposed algorithm, an IEEE 33-bus distribution system $[5,14]$ is adopted and modified by additionally integrating several uncertain sources and DGRs, as shown in Figure 3. Details of the uncertain sources and DGRs are described as follows:

(1) WT, PV, CS and ES stand for wind turbine, photovoltaic module, electric vehicle charging station, and energy storage, respectively. WT, PV, and CS are uncertain sources. ES is regarded as the DGR.

(2) At the moment under study, uncertain sources of the same type are assumed to have the same predicted output or demand. The predicted active power injection to the corresponding bus from WT, PV, and CS are $300 \mathrm{~kW}, 200 \mathrm{~kW}$, and $-500 \mathrm{~kW}$, respectively. The prediction errors of all the uncertain sources are set as $\pm 10 \%$.

(3) The maximum charging or discharging power of ES is assumed to be $\pm 100 \mathrm{~kW}$, and no charging or discharging performance occurs at the moment.

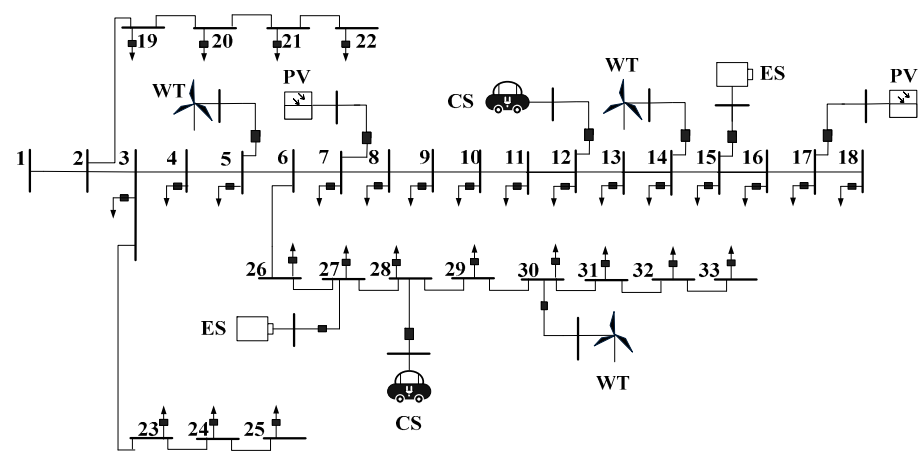

Figure 3. Modified IEEE 33-bus distribution system.

According to the presented regional control strategy, energy storages at buses 15 and 27 are respectively utilized to compensate for the unscheduled power fluctuation. In detail, ES output at bus 15 is adjusted to relieve unscheduled power fluctuation from buses 12,14, and 17; meanwhile, ES output 
at bus 27 is for buses 7, 28, and 30. The interval power flow solutions are shown in Figures 4 and 5, as well as the comparison with scheduled power flow solutions and interval power flow solutions neglecting regional control. Furthermore, the maximum deviation and average deviation among all the buses are illustrated in Table 1, where the deviation specifically refers to the distance from interval power flow solutions' bounds to scheduled power flow solutions.

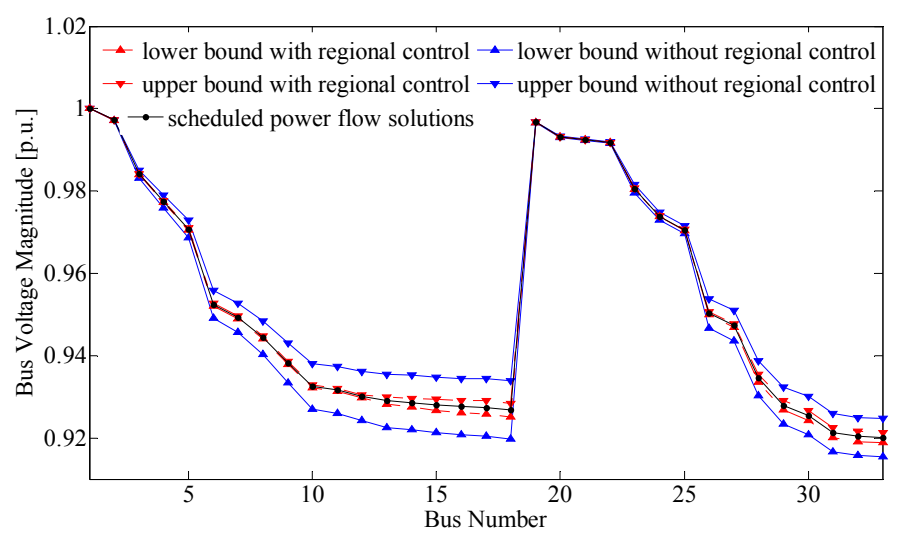

Figure 4. Voltage magnitude bounds in modified IEEE 33-bus distribution system.

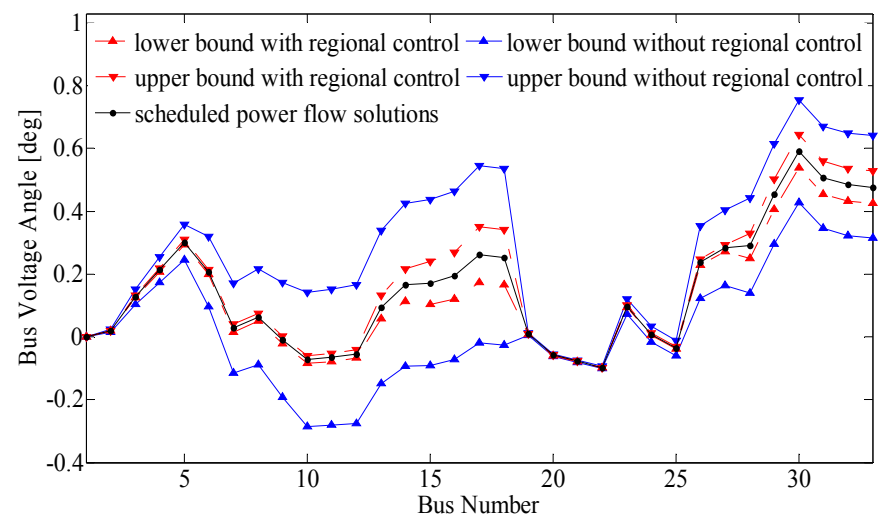

Figure 5. Voltage angle bounds in modified IEEE 33-bus distribution system.

Table 1. Deviation analysis under different scenarios.

\begin{tabular}{ccccc}
\hline \multirow{2}{*}{ Scenario Type } & \multicolumn{2}{c}{ Voltage Magnitude (p.u.) } & \multicolumn{2}{c}{ Voltage Angle (degrees) } \\
\cline { 2 - 5 } & Maximum Deviation & Average Deviation & Maximum Deviation & Average Deviation \\
\hline With regional control & 0.0016 & $5.8667 \times 10^{-4}$ & 0.0882 & $2.5423 \times 10^{-2}$ \\
Without regional control & 0.0070 & $3.5262 \times 10^{-3}$ & 0.2843 & $1.2892 \times 10^{-1}$ \\
\hline
\end{tabular}

Obviously, taking into consideration the presented regional control strategy to smooth the unscheduled power fluctuation, interval power solutions with narrower ranges are obtained. Viewed from voltage magnitude, maximum deviation and average deviation decreased from 0.0070 p.u. to 0.0016 p.u. and $3.5262 \times 10^{-3}$ p.u. to $5.8667 \times 10^{-4}$ p.u., respectively. With respect to voltage angle, maximum deviation and average deviation reduced from 0.2843 degrees to 0.0882 degrees and $1.2892 \times 10^{-1}$ degrees to $2.5423 \times 10^{-2}$ degrees, respectively. Furthermore, the new obtained interval power flow solutions are in good agreement with the practical scenario. Their narrower ranges bring about the elimination of impractical risk warning in power system operations, thereby avoiding unnecessary precautionary measures and reducing the requirement of dispatchable resources. Hence, it is of great significance to propose the affine arithmetic-based power flow algorithm and utilize it to take account of the unscheduled power tracking characteristic of DGRs in power flow analysis. 


\section{Conclusions}

This letter proposes an affine arithmetic-based power flow algorithm, taking into consideration the regional control strategy for unscheduled power fluctuation. Interval power flow solutions obtained by the proposed algorithm exhibit narrower ranges and good agreement with the practical scenario, which will bring significant benefits to the operation of the distribution system. Following this work, more complicated control strategies of DGRs as well as the corresponding affine arithmetic-based modeling methods should be studied, which will make interval power flow solutions more accurate.

Acknowledgments: This work was financially supported by the Scientific Project of Zhejiang Electric Power Company (No. 5211NB160008) and the National Natural Science Foundation of China (No. 51477029).

Author Contributions: All authors contributed to this work. To be specific, Lizi Luo carried out the modeling and calculations. Wei Gu provided the original idea and analyzed the simulation results. Yonghui Wang and Chunxi Chen supplied the original data of case study and revised the manuscript. All authors have read and approved the final manuscript.

Conflicts of Interest: The authors declare no conflict of interest.

\section{References}

1. Yang, N.C. Three-phase power flow calculations by direct $Z_{\mathrm{LOOP}}$ method for microgrids with electric vehicle charging demands. IET Gener. Transm. Distrib. 2013, 7, 1002-1010. [CrossRef]

2. Ou, T.C.; Tsao, T.P.; Lin, W.M.; Hong, C.M.; Lu, K.H.; Tu, C.S. A novel power flow analysis for microgrid distribution system. In Proceedings of the 8th IEEE Conference on Industrial Electronics and Applications (ICIEA), Melbourne, Australia, 19-21 June 2013; pp. 1550-1555.

3. Das, B. Radial distribution system power flow using interval arithmetic. Int. J. Electr. Power Energy Syst. 2002, 24, 827-836. [CrossRef]

4. Vaccaro, A.; Canizares, C.A.; Villacci, D. An affine arithmetic-based methodology for reliable power flow analysis in the presence of data uncertainty. IEEE Trans. Power Syst. 2010, 25, 624-632. [CrossRef]

5. Gu, W.; Luo, L.; Ding, T.; Meng, X.; Sheng, W. An affine arithmetic-based algorithm for radial distribution system power flow with uncertainties. Int. J. Electr. Power Energy Syst. 2014, 58, 242-245. [CrossRef]

6. Jupe, S.C.E.; Taylor, P.C.; Michiorri, A. Coordinated output control of multiple distributed generation schemes. IET Renew. Power Gener. 2010, 4, 283-297. [CrossRef]

7. Pandi, V.R.; Al-Hinai, A.; Feliachi, A. Coordinated control of distributed energy resources to support load frequency control. Energy Convers. Manag. 2015, 105, 918-928. [CrossRef]

8. Pandi, V.R.; Al-Hinai, A.; Feliachi, A. Adaptive coordinated feeder flow control in distribution system with the support of distributed energy resources. Int. J. Electr. Power Energy Syst. 2017, 85, 190-199. [CrossRef]

9. Ou, T.C.; Hong, C.M. Dynamic operation and control of microgrid hybrid power systems. Energy 2014, 66, 314-323. [CrossRef]

10. Ou, T.C. A novel unsymmetrical faults analysis for microgrid distribution systems. Int. J. Electr. Power Energy Syst. 2012, 43, 1017-1024. [CrossRef]

11. Ou, T.C. Ground fault current analysis with a direct building algorithm for microgrid distribution. Int. J. Electr. Power Energy Syst. 2013, 53, 867-875. [CrossRef]

12. Wang, S.; Han, L.; Wu, L. Uncertainty tracing of distributed generations via complex affine arithmetic based unbalanced three-phase power flow. IEEE Trans. Power Syst. 2015, 30, 3053-3062. [CrossRef]

13. Luo, L.; Zhu, J.; Yang, S.; Wang, K.; Yao, J.; Gu, W. Interval Arithmetic based Influence Analysis on Power Flow caused by Integration of Wind Power and Electric Vehicles. In Proceedings of the 19th World Congress of the International Federation of Automatic Control (IFAC), Cape Town, South Africa, $24-29$ August 2014; pp. 2758-2763.

14. Baran, M.E.; Wu, F.F. Network reconfiguration in distribution systems for loss reduction and load balancing. IEEE Trans. Power Deliv. 1989, 4, 1401-1407. [CrossRef]

(C) 2017 by the authors. Licensee MDPI, Basel, Switzerland. This article is an open access article distributed under the terms and conditions of the Creative Commons Attribution (CC BY) license (http://creativecommons.org/licenses/by/4.0/). 